\title{
COEXISTENCE IN REACTION-DIFFUSION SYSTEMS WITH MUTUALIST OR PREDATOR-PREY INTERACTIONS
}

\author{
Walter Kelley
}

\begin{abstract}
The question of the coexistence of two species that exhibit mutualist or predator-prey interactions and satisfy a reaction-diffusion system is studied. It is shown in both cases that under favorable conditions initial populations that are limited in space will never fall below known positive population densities and will eventually extend throughout the potential region of cohabitation.
\end{abstract}

\section{Introduction}

We consider below the asymptotic behavior as $t \rightarrow \infty$ of solutions of reaction-diffusion systems

$$
\begin{aligned}
& u_{t}=\varepsilon^{2} \Delta u+u F(u, v), \\
& v_{t}=\gamma^{2} \Delta v+v G(u, v) .
\end{aligned}
$$

This work is motivated by its potential application to interacting populations, both of which are diffusing in space. The basic question to be addressed is: if the distribution of both species is limited to a small subregion of the potential domain of cohabitation (either through some natural disaster or through the limited introduction of the species into a new domain), under what conditions will the population densities approach some positive coexistence states after a period of time?

The first type of interaction treated here is that of mutually beneficial species. We consider the case where the spatially homogeneous problem has at least two stable steady states: one at the origin and another in the first quadrant. Each population is permitted to satisfy either Dirichlet or Neumann boundary conditions. Hutson [9] and Hutson and Landes [10] provided conditions under which initially localized species in a one-dimensional domain with Neumann boundary conditions approach positive, constant coexistence states. We show that under similar conditions, the populations also will persist in higher dimensional domains. In the case of Dirichlet boundary conditions, we require that the diffusion is relatively slow and obtain coexistence states of boundary-layer type.

In Section 4, we obtain similar results for predator-prey systems. Of course, for such systems the origin is not stable, so we do not expect mutual extinction. In fact, De Mottoni and Rothe [5] have shown that in the case of monotone nullclines and Neumann boundary conditions, a stable steady state in the first quadrant is globally stable. In reality, a species will die out if its population density is too small. Our results show that under favorable conditions, localized predator-prey populations will

Received January 12, 1995, revised June 14, 1995.

1991 Mathematics Subject Classification. 35K57, 92A15.

Key words and phrases: reaction-diffusion system, mutualist and predator-prey interactions, coexistence. 
never fall below a prescribed density and will approach positive coexistence states even in the case that the prey nullcline is not monotone.

Diffusion models for predator-prey interactions with Dirichlet boundary conditions have been studied by Casal et al. [2], Blat and Brown [1], Li [14], Leung [13], LopezGomez and Pardo [15], and others. These papers contain results on the existence and multiplicity of coexistence states. For a survey on coexistence and related issues, see Cosner [4].

\section{Preliminaries}

For the convenience of the reader, we collect here some basic facts about comparison for systems of reaction-diffusion equations and on properties of certain stationary solutions of scalar equations that will be needed to establish our main theorems on coexistence.

First, consider a reaction-diffusion system

$$
w_{t}=D \triangle w+H(w)
$$

where $w: R^{n} \times R^{1} \rightarrow R^{m}, H: R^{m} \rightarrow R^{m}$ is Lipschitz continuous, and $D=\left[\mu_{i j}\right]$ is a diagonal matrix with each $\mu_{i i}>0$. Let $\Omega$ be a smoothly bounded domain in $R^{n}$. At the boundary of $\Omega$, we impose boundary conditions

$$
A w+B \frac{\partial w}{\partial \nu}=0
$$

where $A=\left[a_{i j}\right], B=\left[b_{i j}\right]$ are diagonal matrices with non-negative entries so that $a_{i i}+b_{i i} \neq 0$ for all $i$ and $\frac{\partial}{\partial \nu}$ is the directional derivative in the direction of the outer normal to the boundary of $\Omega$.

Continuous functions $\underline{w}$ and $\bar{w}$ on $\overline{\Omega \times I}, I$ an open interval of real numbers, are called a "subsolution" and a "supersolution", respectively, if (inequalities between vectors indicate component-wise comparisons)

(i) $\underline{w}(x, t) \leq \bar{w}(x, t)$ for $(x, t) \in \overline{\Omega \times I}$,

(ii) $A \underline{w}+B \frac{\partial \underline{w}}{\partial \nu} \leq 0, A \bar{w}+B \frac{\partial \bar{w}}{\partial \nu} \geq 0$ on the boundary of $\Omega$,

(iii) for each point $P$ in $\overline{\Omega \times I}$, there is a ball $B$ about $P$ and finite sets of functions $\left\{\alpha_{i}\right\}$ and $\left\{\beta_{i}\right\}$ on $B$ so that

$$
\begin{aligned}
& \underline{w}^{j}(x, t)=\max _{i} \alpha_{i}^{j}(x, t), \\
& \bar{w}^{j}(x, t)=\min _{i} \beta_{i}^{j}(x, t),
\end{aligned}
$$

for all $(x, t) \in B \cap(\bar{\Omega} \times \bar{I})$, and for each $i, j$,

$$
\begin{gathered}
\alpha_{i t}^{j} \leq \mu_{j j} \Delta \alpha_{i}^{j}+H\left(z^{1}, \cdots, \alpha_{i}^{j}, \cdots, z^{m}\right), \\
\beta_{i t}^{j} \geq \mu_{j j} \Delta \beta_{i}^{j}+H\left(z^{1}, \cdots, \beta_{i}^{j}, \cdots, z^{m}\right),
\end{gathered}
$$

for all $\alpha_{i}^{k} \leq z^{k} \leq \beta_{i}^{k}, k \neq j$.

Theorem 2.1. Assume (2.1) has sub-and supersolutions $\underline{w}$ and $\bar{w}$, respectively. If $w$ is a solution of (2.1) with

$$
\underline{w}(x, 0) \leq w(x, 0) \leq \bar{w}(x, 0) \quad(x \in \bar{\Omega}),
$$

then

$$
\underline{w}(x, t) \leq w(x, t) \leq \bar{w}(x, t)
$$

for all $(x, t) \in \overline{\Omega \times I}$. 
Theorem 2.2. In addition to the hypotheses of Theorem 2.1, assume $\underline{w}$ and $\bar{w}$ are independent of $t$. Then there exists a stationary solution $w_{0}(x)$ so that

$$
\underline{w}(x) \leq w_{0}(x) \leq \bar{w}(x) \quad(x \in \bar{\Omega}) .
$$

Furthermore, every solution $w$ with

$$
\underline{w}(x) \leq w(x, 0) \leq w_{0}(x) \quad(x \in \bar{\Omega})
$$

converges uniformly to $w_{0}(x)$ on $\bar{\Omega}$ as $t \rightarrow \infty$.

Proofs of Theorems 2.1 and 2.2 can be found in Fife and Tang [7]. It also follows from their work that there is a stationary solution, say $w_{1}(x)$, with $w_{1}(x) \geq w_{0}(x)$ on $\bar{\Omega}$ so that every solution starting between the sub- and supersolution must converge to the region bounded by these two stationary solutions. Thus one can conclude that if the stationary solution $w_{0}$ is unique, then all solutions of (2.1) with initial values between the sub- and supersolutions must converge uniformly to $w_{0}$ as $t$ goes to infinity.

Next, consider the stationary scalar equation

$$
\varepsilon^{2} \triangle w+H(w)=0
$$

on a smoothly bounded domain $\Omega$ in $R^{n}$ where $H \in C^{1}(R)$ and $\varepsilon$ is a small, positive parameter. We first assume that $H$ satisfies

(2a) there is a number $z>0$ so that $H(z)=0$ and $H^{\prime}(z)<0$,

(2b) $\int_{q}^{z} H(s) d s>0$ for $0 \leq q<z$.

The following theorem has been proved in various forms by Fife [6], Howes [8], and Clement and Sweers [3]. The variational approach of Clement and Sweers also provides an estimate of how small $\varepsilon$ must be to obtain a solution. The solution often is called a "boundary-layer solution" since it exhibits a rapid change near the boundary of $\Omega$ as $\varepsilon \rightarrow 0$.

Theorem 2.3. Assume $H$ satisfies (2a) and (2b). If $\varepsilon$ is sufficiently small, then (2.2) has a solution $w \geq 0$ so that $w=0$ on $\partial \Omega$, and for some positive constant $C$ (independent of $\varepsilon$ ), $z-C \varepsilon^{2}<w(x)$ whenever $\operatorname{dist}(x, \partial \Omega)>\varepsilon$ and $w(x)<z$ for all $x \in \bar{\Omega}$.

Now we specialize to functions $H$ that satisfy

(2c) there exist $0<z_{1}<z_{2}$ so that $H(0)=H\left(z_{1}\right)=H\left(z_{2}\right)=0, H^{\prime}(0)<0$, $H^{\prime}\left(z_{2}\right)<0$, and $z_{1}$ is the only zero of $H$ between 0 and $z_{2}$.

The proof of the next theorem can be found in Kelley and Ko [12] and Jang [11]. For small values of $\varepsilon$, this theorem yields a "spike layer solution", that is a solution with a narrow spike in the interior of $\Omega$.

Theorem 2.4. Assume $H$ satisfies (2b) (with $z=z_{2}$ ) and (2c). Assume also that $\Omega$ is a ball in $R^{n}$ centered at $x_{0}$. Let $h$ be the unique number less than $z_{2}$ so that $\int_{0}^{h} H(s) d s=0$. Then for sufficiently small values of $\varepsilon,(2.2)$ has a solution $w \geq 0$ so that $w=0$ on the boundary of $\Omega, w$ is radially symmetric with respect to $x_{0}, w$ is decreasing as a function of $\left|x-x_{0}\right|$, there is a $\delta>0$ (independent of $\varepsilon$ ) so that $h+\delta<w\left(x_{0}\right)<z_{2}-\delta$, and for each $C>0, \operatorname{diam}\{w(x)>C\}=O(\varepsilon)(\varepsilon \rightarrow 0)$.

Finally, it will be useful to have two special versions of the "sweeping principle", one for Dirichlet and one for Neumann boundary conditions (see Sattinger [17] and Clement and Sweers [3]). This principle allows one to draw conclusions about the nature of positive solutions of semilinear equations and systems of equations. 
Theorem 2.5. Let $\alpha_{s}(x)$ be a one-parameter family of $C^{2}$ functions for $0 \leq s \leq 1$ so that

(a) $\bar{D}_{s} \subset \Omega$ where $D_{s} \equiv\left\{x: \alpha_{s}(x)>0\right\},(0 \leq s \leq 1)$,

(b) $0 \leq \Delta \alpha_{s}+H\left(\alpha_{s}\right)$ in $D_{s}(0 \leq s \leq 1), H(0) \geq 0$,

(c) the function $s \mapsto \max \left\{0, \alpha_{s}\right\}$ is continuous from $[0,1]$ to $C(\bar{\Omega})$.

For each $u \in C^{2}(\Omega) \cap C(\bar{\Omega})$ that satisfies

$$
\begin{gathered}
\triangle u+H(u) \leq 0 \quad(x \in \Omega), \\
u>0 \quad(x \in \Omega), \\
u>\alpha_{0} \quad\left(x \in D_{0}\right),
\end{gathered}
$$

we have $u>\alpha_{s}$ in $D_{s}$ for $0 \leq s \leq 1$.

Proof. Let

$$
\begin{aligned}
I & \equiv\left\{s \in[0,1]: u>\alpha_{s} \text { on } D_{s}\right\} \\
& =\left\{s \in[0,1]: u>\max \left\{\alpha_{s}, 0\right\} \text { on } \Omega\right\} .
\end{aligned}
$$

Now $0 \in I$ and $I$ is open in $[0,1]$. Let $\delta$ be the largest number so that $[0, \delta) \subset I$. We claim $\delta \in I$. If not, there is an $\bar{x}$ in $D_{\delta}$ so that $u(\bar{x})=\alpha_{\delta}(\bar{x})$. By the maximum principle and the hypotheses, it is impossible that $\alpha_{\delta}(x) \leq u(x)$ for all $x \in D_{\delta}$. Thus $u(x)<\alpha_{\delta}(x)$ for some $x \in D_{\delta}$, contradicting the choice of $\delta$. We conclude that $\delta \in I$. Thus $I=[0,1]$.

Theorem 2.6. Let $\alpha_{s}(x)$ be a one-parameter family of $C^{2}$ functions for $0 \leq s \leq 1$ so that

(a) $\frac{\partial \alpha_{s}}{\partial \nu}<0$ at each point in $D_{s} \cap \partial \Omega(0 \leq s \leq 1)$,

(b) $0 \leq \triangle \alpha_{s}+H\left(\alpha_{s}\right)$ in $D_{s}(0 \leq s \leq 1), H(0) \geq 0$,

(c) the function $s \mapsto \max \left\{0, \alpha_{s} \mid \bar{\Omega}\right\}$ is continuous from $[0,1]$ to $C(\bar{\Omega})$.

For any $u \in C^{2}(\Omega) \cap C(\bar{\Omega})$ that satisfies

$$
\begin{gathered}
\Delta u+H(u) \leq 0 \quad(x \in \Omega), \\
u \geq 0 \quad(x \in \Omega), \\
\frac{\partial u}{\partial \nu} \geq 0 \quad(x \in \partial \Omega), \\
u>\alpha_{0} \quad\left(x \in D_{0} \cap \Omega\right),
\end{gathered}
$$

we have $u>\alpha_{s}$ on $D_{s} \cap \Omega$ for $0 \leq s \leq 1$.

Proof. Let $I \equiv\left\{s \in[0,1]: u>\alpha_{s}\right.$ on $\left.D_{s} \cap \Omega\right\}$. Now if $u>\max \left\{\alpha_{s}, 0\right\}$ in $\Omega$ and if $u(x)=\alpha_{s}(x)$ for some $x \in \partial \Omega$, then the strong maximum principle implies that $\frac{\partial}{\partial \nu}\left(u-\alpha_{s}\right)<0$ at $x$. This contradiction allows us to conclude that $I$ is open in $[0,1]$. The rest of the proof is similar to that of Theorem 2.5.

\section{A model of mutualism}

In this section, $u$ and $v$ will denote densities of species that interact in a mutually beneficial way and that diffuse throughout some spatial domain $\Omega \subset R^{n}$. The model will have the form

$$
\begin{aligned}
& u_{t}=\varepsilon^{2} \triangle u+u F(u, v), \\
& v_{t}=\gamma^{2} \triangle v+v G(u, v),
\end{aligned}
$$


where $\varepsilon$ and $\gamma$ represent positive parameters. We consider only the case that the spatially homogeneous problem

$$
\begin{aligned}
& u_{t}=u F(u, v), \\
& v_{t}=v G(u, v),
\end{aligned}
$$

has stable steady states at the origin and at a point $\left(u_{1}, v_{1}\right)$ in the first quadrant, as well as an intermediate unstable steady state $\left(u_{0}, v_{0}\right)$. Thus we assume that both species are obligate, i.e., unable to survive in the absence of the other. Typical nullclines are shown in Figure 1.

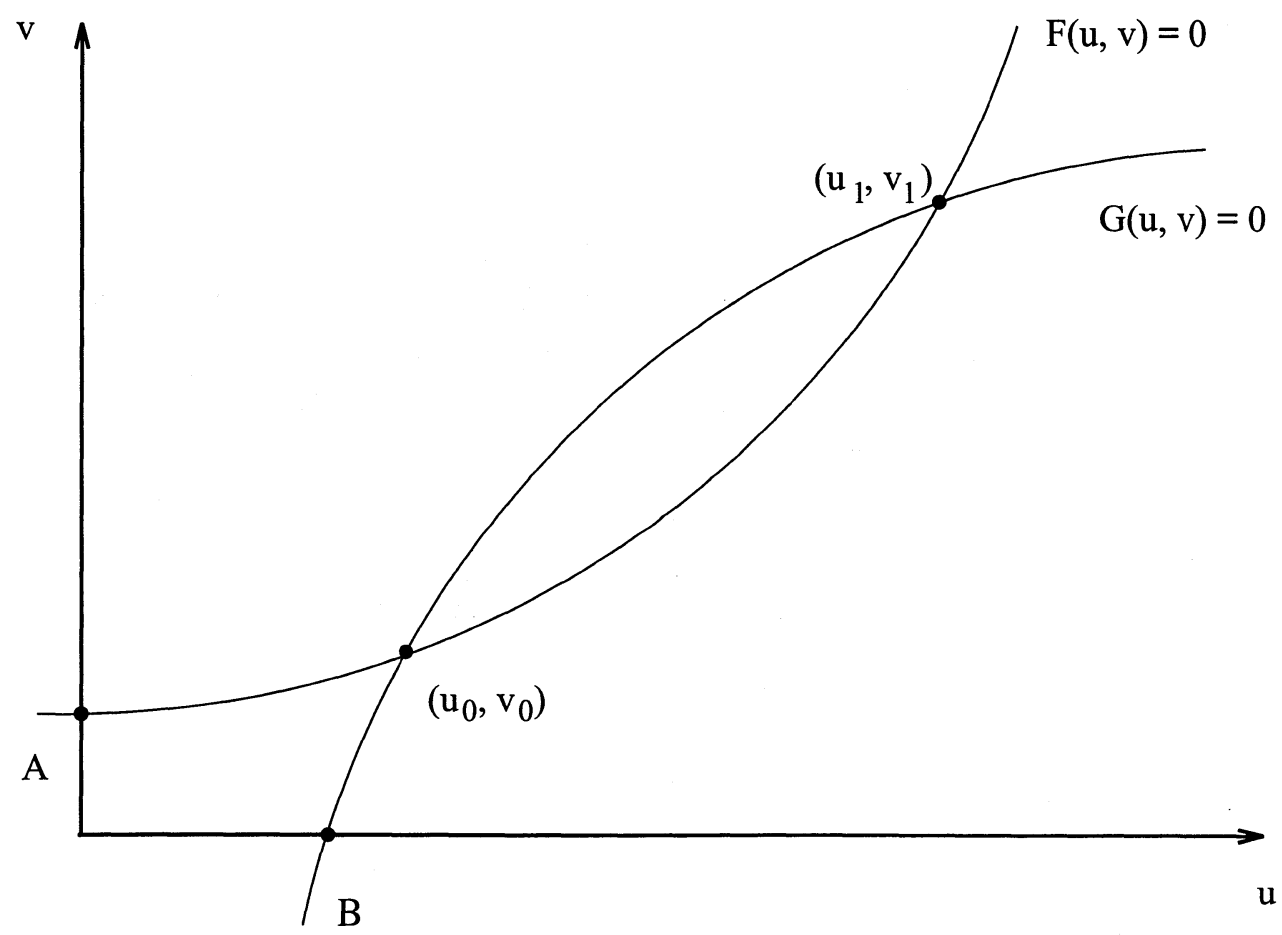

Figure 1. Typical nullclines.

In order to describe this interaction and ensure coexistence of the species, we make the assumptions

(3a) $\frac{\partial F}{\partial u}<0, \frac{\partial F}{\partial v}>0$ for $u, v>0$,

(3b) $\frac{\partial G}{\partial u}>0, \frac{\partial G}{\partial v}<0$ for $u, v>0$,

(3c) the curves $F=0$ and $G=0$ intersect in exactly two points $\left(u_{0}, v_{0}\right)$ and $\left(u_{1}, v_{1}\right)$ with $0<u_{0}<u_{1}$ and $0<v_{0}<v_{1}, F=0$ intersects the $v$-axis at $A>0$, and $G=0$ intersects the $u$-axis at $B>0$,

(3d) there is a $C^{2}$ curve $(\hat{u}(w), \hat{v}(w)), 0 \leq w \leq 1$, so that $\hat{u}(0)=\hat{v}(0)=0, \hat{u}(1)=u_{1}$, $\hat{v}(1)=v_{1}, \hat{u}^{\prime}, \hat{v}^{\prime}>0, \hat{u}^{\prime \prime}, \hat{v}^{\prime \prime} \geq 0$, and an $h \in C^{1}[0,1]$ so that

$$
h(w) \leq \min \left\{\frac{\left(\varepsilon^{2}+\gamma^{2}\right) \hat{u}(w) F(\hat{u}(w), \hat{v}(w))}{\varepsilon^{2} \hat{u}^{\prime}(w)}, \frac{\left(\varepsilon^{2}+\gamma^{2}\right) \hat{v}(w) G(\hat{u}(w), \hat{v}(w))}{\gamma^{2} \hat{v}^{\prime}(w)}\right\}
$$

and $\int_{w}^{1} h(\tau) d \tau>0$ for $0 \leq w<1$. 
These hypotheses are similar to those employed by Hutson [9] for the case of one spatial dimension. We refer the reader to his paper and the references contained therein for further discussion. Although we are considering only one of several possible types of mutualist interactions, the remaining cases are easily treated by the same methods.

Theorem 3.1. Assume hypotheses (3a)-(3d). Let $x_{0} \in \Omega$. If $\varepsilon$ and $\gamma$ are sufficiently small, then there is a continuous function $\phi(x)$ on $\Omega$ with the properties

(a) $\phi(x) \geq 0$, for each $C>0, \operatorname{diam}\{x: \phi(x)>C\}=O(\varepsilon+\gamma)$,

(b) $\max _{x \in \Omega} \phi(x)=\phi\left(x_{0}\right) \in\left(w_{0}, 1\right)$ where $\int_{0}^{w_{0}} h(\tau) d \tau=0$,

so that the graph of each solution $(u, v)$ of $(3.1)$ that satisfies $u(x, 0)>\hat{u}(\phi(x))$, $v(x, 0)>\hat{v}(\phi(x)), x \in \Omega$, and Dirichlet or Neumann boundary conditions on $\partial \Omega$ converges as $t \rightarrow \infty$ to an order interval

$$
\left\{(x, \alpha, \beta): U(x) \leq \alpha \leq u_{1}, V(x) \leq \beta \leq v_{1}\right\}
$$

where $(U, V)$ is a pair of stationary solutions that satisfy the same boundary conditions as $u$ and $v$, and

$$
\hat{u}\left(w_{0}\right)<U(x), \quad \hat{v}\left(w_{0}\right)<V(x)
$$

for $x \in \Omega$ (Neumann boundary conditions) or for $\operatorname{dist}(x, \partial \Omega)>C_{1} \varepsilon$ (Dirichlet boundary conditions) where $C_{1}$ is a positive constant.

Proof. Choose a ball $B_{r}$ about $x_{0}$ so that $B_{r} \subset \Omega$. By Theorem 2.4, there is a continuous function $\phi$ that satisfies:

$$
\left(\varepsilon^{2}+\gamma^{2}\right) \triangle \phi+h(\phi)=0
$$

in $B_{r}$ for small values of $\delta>0$, as well as properties (a) and (b) for small values of $\varepsilon$ and $\gamma$, and $\phi$ is symmetric with respect to $x_{0}$, decreasing in $\left|x-x_{0}\right|$, and $\phi=0$ outside of $B_{r}$.

We claim that $(\underline{u}, \underline{v})=(\hat{u}(\phi), \hat{v}(\phi))$ is a subsolution for (3.1). Note that

$$
\begin{aligned}
\varepsilon^{2} \Delta \underline{u}+\underline{u} F(\underline{u}, \underline{v}) & \geq \varepsilon^{2}\left[\hat{u}^{\prime}(\phi) \Delta \phi+\hat{u}^{\prime \prime}(\phi) \sum_{i} \phi_{x_{i}}^{2}\right]+\frac{\varepsilon^{2}}{\varepsilon^{2}+\gamma^{2}} h(\phi) \hat{u}^{\prime}(\phi) \\
& \geq \frac{\varepsilon^{2}}{\varepsilon^{2}+\gamma^{2}} \hat{u}^{\prime}(\phi)\left[\left(\varepsilon^{2}+\gamma^{2}\right) \triangle \phi+h(\phi)\right] \\
& =0
\end{aligned}
$$

if $\varepsilon$ is sufficiently small. A similar calculation shows that

$$
\gamma^{2} \triangle \underline{v}+\underline{v} G(\underline{u}, \underline{v}) \geq 0
$$

when $\gamma$ is small.

Now let $(u, v)$ be a solution pair for (3.1) with $u(x, 0)>\hat{u}(\phi(x)), v(x, 0)>\hat{v}(\phi(x))$ $(x \in \Omega)$. Let $\bar{u}(t), \bar{v}(t)$ be spatially homogeneous solutions so that

$$
\bar{u}(0)=\max \left\{u_{1}, \max _{x \in \bar{\Omega}} u(x, 0)\right\}, \quad \bar{v}(0)=\max \left\{v_{1}, \max _{x \in \bar{\Omega}} v(x, 0)\right\} .
$$

Then $\bar{u}, \bar{v}$ are supersolutions for $(3.1)$ and $\bar{u}(t) \rightarrow u_{1}, \bar{v}(t) \rightarrow v_{1}$ as $t \rightarrow \infty$. From Theorem 2.1,

$$
\hat{u}(\phi(x)) \leq u(x, t) \leq \bar{u}(t), \quad \hat{v}(\phi(x)) \leq v(x, t) \leq \bar{v}(t)
$$

for all $x \in \bar{\Omega}, t \geq 0$. We can use Theorem 2.2 (and the remarks following) to obtain positive stationary solutions $U$ and $V$ so that the graph of $(u, v)$ converges as $t \rightarrow \infty$ to the order interval $\left\{(x, \alpha, \beta): U(x) \leq \alpha \leq u_{1}, V(x) \leq \beta \leq v_{1}\right\}$. 
Finally, we verify the lower bound on $U$ for the case of Dirichlet boundary conditions. The arguments for $V$ and for Neumann boundary conditions are similar. Now the subsolution $\underline{u}$ defined above is of class $C^{2}$ where it is positive. If we restrict $\underline{u}$ to the set where it is positive, then one-parameter families of subsolutions can be defined by translation along curves $r(s): R^{1} \rightarrow \Omega$ with $r(0)=x_{0}$ by

$$
\alpha_{s}(x)=\underline{u}(x-r(s)) .
$$

Then for each $s, \alpha_{s}$ satisfies

$$
\varepsilon^{2} \triangle \alpha_{s}+\alpha_{s} F\left(\alpha_{s}, V\right) \geq \varepsilon^{2} \triangle \alpha_{s}+\alpha_{s} F\left(\alpha_{s}, \underline{v}\right) \geq 0 .
$$

It follows from Theorem 2.5 that $U(x)>\alpha_{s}(x)$ as long as $\left\{x: \alpha_{s}(x)>0\right\} \subset \Omega$ and hence from the properties of $\phi$ that $U(x)>\hat{u}\left(w_{0}\right)$ if $\operatorname{dist}(x, \partial \Omega)>C_{1} \varepsilon$ for some $C_{1}>0$.

Note that the estimates on $U$ and $V$ imply that in the case of Dirichlet boundary conditions, the coexistence states are of boundary-layer type. In the case of Neumann boundary conditions, it is likely that the coexistence states are constants, but we do not know how to prove this except for the case of one spatial dimension (see Hutson [9]).

If both populations satisfy Neumann boundary conditions, slow diffusion is not essential for coexistence. However, we do need to assume that the initial populations are spread across a relatively larger subregion of $\Omega$.

Theorem 3.2. Assume hypotheses (3a)-(3d). Let $x_{0} \in \Omega$ and let $B_{r}$ be a ball about $x_{0}$ of sufficiently large radius $r$. Then there is a continuous function $\phi$ with the properties

(a) $\phi(x) \geq 0, \phi(x)=0$ outside of $B_{r}$, for each $C>0, \operatorname{diam}\{x: \phi(x)>C\}$ has a bound that is independent of $r$,

(b) $\max _{x \in \Omega} \phi(x)=\phi\left(x_{0}\right) \in\left(w_{0}, 1\right)$ where $\int_{0}^{w_{0}} h(\tau) d \tau=0$,

so that the graph of each solution $(u, v)$ of (3.1) that satisfies $u(x, 0)>\hat{u}(\phi(x))$, $v(x, 0)>\hat{v}(\phi(x)), x \in \Omega$, and Neumann boundary conditions on $\partial \Omega$, converges as $t \rightarrow \infty$ to an order interval

$$
\left\{(x, \alpha, \beta): U(x) \leq \alpha \leq u_{1}, V(x) \leq \beta \leq v_{1}\right\}
$$

where $(U, V)$ is a pair of stationary solutions that satisfy Neumann boundary conditions, and

$$
\hat{u}\left(w_{0}\right)<U(x), \quad \hat{v}\left(w_{0}\right)<V(x)
$$

for $x \in \Omega$.

Proof. The proof follows the outline of the proof of Theorem 3.1. To obtain $\phi$, we rescale by the change of variable $z=x / r$; then $\phi$ is to be a solution of

$$
\frac{\varepsilon^{2}+\gamma^{2}}{r^{2}} \triangle \phi+h(\phi)=0 .
$$

Theorem 2.4 can be applied (for large enough $r$ ) to obtain a $\phi$ with the desired properties, and $\underline{u}$ and $\underline{v}$ are defined as in the proof of Theorem 3.1. Now $B_{r} \cap \partial \Omega$ may not be empty, but since $\underline{u}$ and $\underline{v}$ are radially symmetric and decreasing as functions of the radial variable, we must have $\frac{\partial \underline{u}}{\partial \nu} \leq 0$ and $\frac{\partial \underline{v}}{\partial \nu} \leq 0$ at $\partial \Omega$. The remainder of the proof is similar to that of Theorem 3.1 . 


\section{Predator-prey problems}

In this section, we consider models for the interaction of a prey population with density $u(x, t)$ and a predator population with density $v(x, t)$. If both populations are diffusing in space, then we have as in the preceding section

$$
\begin{aligned}
& u_{t}=\varepsilon^{2} \triangle u+u F(u, v), \\
& v_{t}=\gamma^{2} \triangle v+v G(u, v) .
\end{aligned}
$$

The spatial domain again is denoted by $\Omega \subset R^{n}$, and it is assumed to be smoothly bounded.

We are interested in studying the coexistence of predator and prey for the case that the spatially homogeneous problem

$$
\begin{aligned}
u_{t} & =u F(u, v), \\
v_{t} & =v G(u, v)
\end{aligned}
$$

has a stable steady state in the first quadrant. The nullclines then might be as pictured in Figure 2.

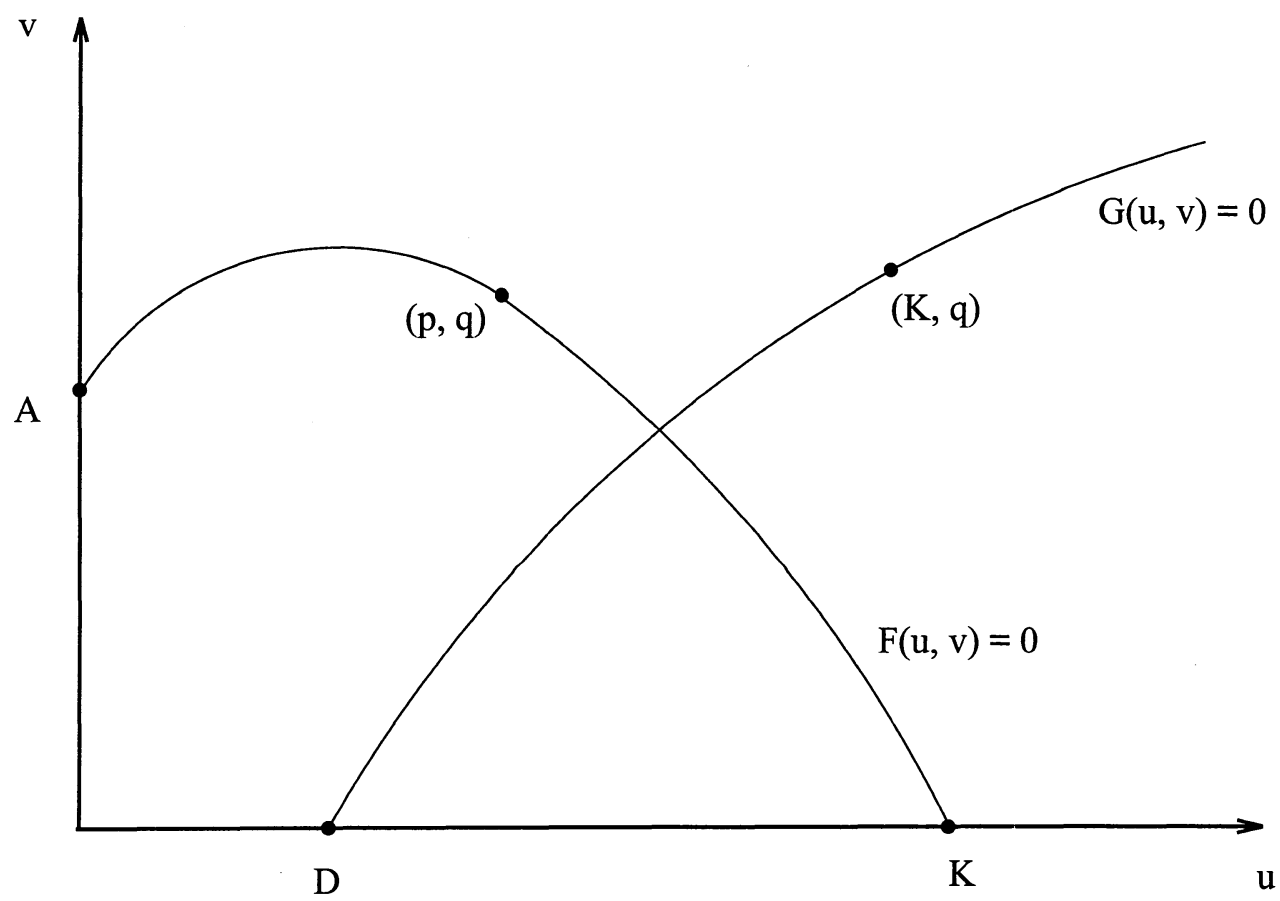

FIGURE 2. Nullclines for predator-prey problems.

Specifically, we assume

(4a) $\frac{\partial F}{\partial v}<0, \frac{\partial G}{\partial u}>0$, for $u, v>0$,

(4b) the curves $F=0$ and $G=0$ intersect in a point in the first quadrant where the slope of $F=0$ is negative,

(4c) the curve $F=0$ intersects the $v$-axis at $A \geq 0$ and the $u$-axis at $K>0$,

(4d) the curve $G=0$ intersects the $u$-axis at $0 \leq D<K$ and $\frac{\partial G}{\partial v}<0$ along this curve. 
The preceding assumptions, as well as the type of nullclines illustrated in Figure 2, are classical for predator-prey models (see Murray [16]). They are based on the following assumptions: the density of predators needed to maintain a constant density of prey (for a spatially homogeneous population) is positive for small prey density and decreases to 0 at the carrying capacity of the prey. The density of prey required to maintain a constant density of predators is positive and increasing for an increasing density of predators.

For our discussion, we need two additional quantities. Let $q$ be the unique value of $v$ so that $G(K, q)=0$ (thus $q$ is the number of predators maintained if the prey population is held at its carrying capacity). Now assume

(4e) there is a value $p \in(D, K)$ so that $F(p, q)=0, \frac{\partial F}{\partial u}(p, q)<0$, and $\int_{u}^{p} s F(s, q) d s>0$ for $0 \leq u<p$.

Hypothesis (4e) is the central condition needed to establish the coexistence of predator and prey populations, assuming sufficient start-up population densities. It requires that the average rate of change of prey population density is positive over certain population intervals, given that the predator population density is equal to $q$.

In our first theorem, the predator and prey populations may satisfy either Dirichlet or Neumann boundary conditions. It establishes that if diffusion of predator and prey is relatively slow, then initial populations confined to $B_{r}$, a ball of radius $r$ inside the domain, will stabilize over time. How slow the diffusion must be depends on the size of $r$, and in fact, an elementary scaling argument shows that $\varepsilon$ and $\gamma$ are inversely proportional to $r$.

Theorem 4.1. Assume hypotheses (4a)-(4e). Let $B_{r} \subset \Omega$. If $\varepsilon$ and $\gamma$ are sufficiently small, then there are continuous functions $\underline{u}(x)$ and $\underline{v}(x)$ with the properties

(a) $p>\underline{u}(x)>p-C \varepsilon^{2}$ for $x \in B_{r-\varepsilon}$ and some constant $C$ independent of $\varepsilon$, $\underline{u}(x)=0$ for $x \notin B_{r}$,

(b) $w>\underline{v}(x)>0$ for $x \in B_{r-\varepsilon}$ where $w$ satisfies $G(p, w)=0, \underline{v}(x)=0$ for $x \notin B-r$, so that the graph of each solution $(u, v)$ of (4.1) that satisfies $K>u(x, 0)>\underline{u}(x)$, $q>v(x, 0)>\underline{v}(x), x \in \Omega$, and Dirichlet or Neumann boundary conditions, also satisfies these inequalities for all $t>0$ and converges as $t \rightarrow \infty$ to an order interval

$$
\left\{(x, \phi, \theta): u_{1}(x) \leq \phi \leq u_{2}(x), v_{1}(x) \leq \theta \leq v_{2}(x)\right\}
$$

where $\left(u_{1}, v_{1}\right)$ and $\left(u_{2}, v_{2}\right)$ are positive stationary solutions that satisfy the same boundary conditions as $(u, v)$, and there are positive constants $C_{1}, C_{2}, C_{3}$ so that

$$
p-C_{1} \varepsilon^{2}<u_{1}(x), \quad w-C_{2} \varepsilon^{2}<v_{1}(x)
$$

for $x \in \Omega$ (Neumann boundary conditions) or for $\operatorname{dist}(x, \partial \Omega)>C_{3} \varepsilon$ (Dirichlet boundary conditions).

Proof. First, note that $\bar{u}=K, \bar{v}=q$ constitute a supersolution pair for (4.1) since

$$
\bar{u}_{t}-\varepsilon \triangle \bar{u}=0 \geq \bar{u} F(\bar{u}, v)
$$

for all $0 \leq v \leq \bar{v}$, and

$$
\bar{v}_{t}-\gamma \triangle \bar{v}=0=\bar{v} G(\bar{u}, \bar{v}) .
$$

By Theorem 2.3 and hypothesis (4e), there is for small values of $\varepsilon$ a function $\tilde{u}(x)$ having the properties

(i) $\tilde{u}=0$ on $\partial B_{r}$

(ii) $\tilde{u}>p-C_{1} \varepsilon^{2}$ for $x \in B_{r-\varepsilon}$ and some $C_{1}>0$,

(iii) $\tilde{u}<p$ on $B_{r}$, 
(iv) $\tilde{u}_{t}-\varepsilon^{2} \triangle \tilde{u}=-\varepsilon^{2} \triangle \tilde{u} \leq \tilde{u} F(\tilde{u}, q)$ for $x \in B_{r}$.

Define

$$
\underline{u}= \begin{cases}\tilde{u}(x) & \text { for } x \in B_{r} \\ 0 & \text { for } x \in \bar{\Omega}-B_{r} .\end{cases}
$$

Then it follows easily that $\underline{u}$ is a subsolution for (4.1).

If $\varepsilon$ is small, then $p-C_{1} \varepsilon^{2}>D$ by assumption (4e), so $G\left(p-C_{1} \varepsilon^{2}, 0\right)>0$. Again by Theorem 2.3, for small values of $\gamma$ there is a function $\tilde{v}(x)$ so that

(i) $\tilde{v}=0$ on $\partial B_{r-\varepsilon}$,

(ii) $w-C_{2} \varepsilon^{2}<\tilde{v}<w$ in $B_{r-\varepsilon}$ for some $C_{2}>0$,

(iii) $\tilde{v}_{t}-\gamma^{2} \triangle \tilde{v}=-\gamma^{2} \triangle \tilde{v} \leq \tilde{v} G\left(p-C_{1} \varepsilon^{2}, \tilde{v}\right) \leq \tilde{v} G(\tilde{u}, \tilde{v})$

by assumption (3a) for $x$ in $B_{r-\varepsilon}$. Thus

$$
\underline{v}(x)= \begin{cases}\tilde{v}(x) & \text { for } x \in B_{r-\varepsilon} \\ 0 & \text { for } x \in \bar{\Omega}-B_{r-\varepsilon}\end{cases}
$$

is a subsolution. The behavior of solutions of (4.1) described in the statement of the theorem now follows immediately from Theorem 2.2, and the properties of $u_{1}$ and $v_{1}$ are verified as in the proof of Theorem 3.1.

For certain special systems of the form (4.1) with Dirichlet boundary conditions, it is known that positive steady-state solutions are unique (see Lopez-Gomez and Pardo [15] and Casal et al. [2]). Then solutions of the reaction-diffusion system described in Theorem 4.1 must converge to the steady-state solution as $t \rightarrow \infty$.

In the case that both populations satisfy Neumann boundary conditions, we have a result for predator-prey models similar to Theorem 3.2.

Theorem 4.2. Assume hypotheses (4a)-(4e). If $r$ is large enough, then there are continuous functions $\underline{u}(x), \underline{v}(x)$ with the properties

(a) $p>\underline{u}(x)>p-\frac{C}{r^{2}}$ for $x \in B_{r-s} \cap \Omega$ (s,C independent of $r$ ), $\underline{u}(x)=0$ for $x \in \Omega \backslash B_{r}$,

(b) $w>\underline{v}(x)>0$ for $x \in B_{r-s} \cap \Omega, \underline{v}(x)=0$ for $x \in \Omega \backslash B_{r}$,

so that the graph of each solution $(u, v)$ of (4.1) that satisfies $K>u(x, 0)>\underline{u}(x)$, $q>v(x, 0)>\underline{v}(x) x \in \Omega$, and Neumann boundary conditions, also satisfies these inequalities for all $t>0$ and converges as $t \rightarrow \infty$ to an order interval

$$
\left\{(x, \phi, \theta): u_{1}(x) \leq \phi \leq u_{2}(x), v_{1}(x) \leq \theta \leq v_{2}(x)\right\}
$$

where $\left(u_{1}, v_{1}\right)$ and $\left(u_{2}, v_{2}\right)$ are stationary solutions of (4.2) satisfying Neumann boundary conditions, and there are constants $C_{1}, C_{2}$ so that

$$
p-\frac{C_{1}}{r^{2}}<u_{1}(x) \leq u_{2}(x)<K, w-\frac{C_{2}}{r^{2}}<v_{1}(x) \leq v_{2}(x)<q
$$

for all $x \in \Omega$.

This result is easily obtained by modifying the proof of Theorem 4.1, as the proof of Theorem 3.1 was modified to obtain Theorem 3.2.

\section{References}

1. J. Blat and K. Brown, Global bifurcations of positive solutions in some systems of elliptic equations, SIAM J. Math. Anal. 17 (1986), 1339-1353.

2. A. Casal, J. Eilbeck, and J. Lopez-Gomez, Existence and uniqueness of coexistence states for a predator-prey model with diffusion, Diff. Int. Eqns. 7 (1994), 411-440. 
3. P. Clement and G. Sweers, Existence and multiplicity results for a semilinear elliptic eigenvalue problem, Ann. Scuola Normale Superior Pisa 14 (1987), 97-121.

4. C. Cosner, Persistance (Permanance), compressivity, and practical persistance in some reactiondiffusion models from ecology. In: Comparison Methods and Stability Theory (X. Liu and D. Siegel, eds.), Dekker, New York, 1994, pp.101-114.

5. P. De Mottoni and F. Rothe, Convergence to homogeneous equilibrium states for generalized Volterra-Lotka systems, SIAM J. Appl. Math 37(1979), 648-663.

6. P. Fife, Semilinear elliptic boundary value problems with small parameters, Arch. Rat. Mech. Anal. 52 (1973), 205-232.

7. P. Fife and M. Tang, Comparison principles for reaction-diffusion systems: irregular comparison functions and applications to questions of stability and speed of propagation of disturbances, J. Diff. Eqns. 40 (1981), 168-185.

8. F. Howes, Boundary-interior layer interactions in nonlinear singular perturbation theory, Mem. AMS 15 (1978).

9. V. Hutson, Stability in a reaction-diffusion model of mutualism, SIAM J. Math. Anal. 17 (1986), $58-66$.

10. V. Hutson and R. Landes, Phragmen-Lindelof principle for weak subsolutions and cooperative reaction-diffusion systems, Appl. Anal. 54 (1994), 181-193.

11. J. Jang, On spike solutions of singularly perturbed semilinear Dirichlet problems, J. Diff. Eqns. 114 (1994), 370-395.

12. W. Kelley and B. Ko, Semilinear elliptic singular perturbation problems with nonuniform interior behavior, J. Diff. Eqns. 86 (1990), 88-101.

13. A. Leung, Monotone schemes for semilinear elliptic systems related to ecology, Math. Meth. Appl Sci. 4 (1982), 272-285.

14. L. Li, Coexistence theorems of steady states for predator-prey interacting systems, Trans. AMS 305 (1988), 143-166.

15. J. Lopez-Gomez and R. Pardo, Existence and uniqueness of coexistence states for the predatorprey Lotka-Volterra model with diffusion on intervals, Diff. Int. Eqns. 6 (1993), 1025-1031.

16. J. Murray, Mathematical Biology, Springer-Verlag, 1989.

17. D. Sattinger, Topics in Stability and Bifurcation Theory, Lecture Notes in Math 309, SpringerVerlag, 1973.

Department of Mathematics, University of Oklahoma, Norman, OK 73019, USA 